\author{
MAGDALENA SOWA \\ Uniwersytet Marii Curie-Skłodowskiej w Lublinie \\ magdalena.sowa@umcs.pl \\ ORCID: 0000-0002-9571-8693
}

\title{
Défis méthodologiques de la programmation des cours en langues sur objectifs spécifiques
}

\section{Some methodological challenges of designing LSP courses}

\begin{abstract}
The aim of the text is to discuss issues related to planning LSP courses. The key stages of course design are understandable and clear to most LSP teachers. However, their practical implementation can raise certain doubts or questions. The paper attempts to analyse such problematic aspects of LSP course planning in detail. We will situate these problems at particular stages during course design and show to what extent they can affect the success of LSP education. In our opinion, the diagnosis and analysis of these sensitive areas can be helpful not only in the effective planning of LSP training programmes but also in language teachers' education for professional purposes.
\end{abstract}

KEYWORDS: Language for specific purposes, course design, needs analysis, LSP teacher, source data, designing activities.

MOTS-CLÉS: Langue sur objectifs spécifiques, programmation de formation, enseignant de LOS, données du terrain, élaboration des activités.

Tout enseignant de langue sur objectifs spécifiques (désormais LOS) doit se confronter à une suite de tâches de conception et de programmation afin de mettre en place une formation linguistico-professionnelle réussie. Si l'accomplissement de ces actions se situe dans la phase précédant la réalisa- 
tion réelle des cours de langue, elles exercent une influence décisive sur l'adéquation de la forme et du contenu de ces derniers par rapport à la satisfaction des publics cibles et déterminent le succès final de l'enseignement. Il en résulte que même si le modèle prototypique de l'élaboration des programmes de formation linguistique sur objectifs spécifiques suggère en grandes lignes les étapes et les actions à accomplir par l'enseignant/ concepteur, ces dernières ne sont pas exemptes de zones de doutes et/ou dilemmes méthodologiques qui tourmentent les concepteurs visant la mise en place des cours de langue de spécialité ${ }^{1}$ pour des publics professionnels particuliers. Une telle constatation contraint à s'interroger plus en détail sur la nature des difficultés potentielles (et invisibles au premier abord) qui résultent des étapes successives de la planification des cours et auxquelles les enseignants / concepteurs sont obligés d'apporter une solution pédagogique pertinente.

La réflexion sur des aspects plus ou moins problématiques de la planification des formations en langues de spécialité (qu'il est aussi possible d'envisager en termes de défis méthodologiques qui se présentent à l'enseignant / concepteur) constitue l'objectif majeur du présent texte. La discussion des questions particulières à résoudre au cours du travail conceptuel de l'enseignant sera précédée d'un bref rappel du modèle de programmation des formations en langues de spécialité. La mise en relation des étapes que l'enseignant réalise afin de construire une proposition de cours réussie avec des difficultés (ou les zones de turbulence) censées accompagner son travail peut s'avérer bénéfique pour la réflexion ultérieure sur les modalités de la formation des enseignants de langues de spécialité.

\section{1. ÉLABORER LA FORMATION EN LANGUES DE SPÉCIALITÉ : PRINCIPES MÉTHODOLOGIQUES DE RÉFÉRENCE}

La didactique des langues sur objectifs spécifiques vise à décrire, expliciter et programmer le processus d'acquisition des savoir-faire linguistiques spécialisés (S. Grucza 2007 : 11). Seule la programmation attire l'attention sur le rôle-clé qu'y joue l'enseignant de langue, ce dernier ayant une influence décisive sur la forme finale du programme d'enseignement. Programmer la formation en LOS est une activité ardue: sa décomposition en plusieurs étapes requiert de l'investissement en temps, la quantité du travail à réaliser

${ }^{1}$ Dans le cadre du présent texte, nous considérons les termes «langue(s) sur objectifs spécifiques », « langue(s) de spécialité », « langue(s) à des fins professionnelles » comme synonymes. 
exige un engagement personnel important, la coopération avec les tiers incite à interagir avec divers acteurs du terrain, la concentration sur la conception de la formation entière et sa réalisation matérielle contraignent à une activité réflexive permanente. C'est aussi un processus à long terme, envisagé à travers plusieurs actions qui se succèdent dans le temps et doivent être accomplies au moment précis et en adéquation avec les objectifs de la formation visés. L'essentiel de la programmation consiste à recenser les contenus à enseigner et les agencer de manière à permettre aux apprenants d'acquérir et/ou de développer les savoirs et savoir-faire étant à la base de la demande de formation.

Le modèle d'élaboration des formations en langues sur objectifs spécifiques que nous considérons comme prototypique est celui de Mangiante et Parpette (2004). Conçu en principe pour l'enseignement du Français sur Objectifs Spécifiques (FOS), il s'applique aisément aux formations dédiées à toute autre langue. Les actions accomplies par l'enseignant / concepteur au cours de cinq étapes consistent progressivement à identifier la demande, analyser les besoins du public cible, réunir les données, analyser le corpus et élaborer les activités pédagogiques. Au fur et à mesure de la programmation prescrite par le modèle en question, l'enseignant / concepteur prend connaissance des motifs de la demande de formation et des besoins qu'il importe de satisfaire par le programme de formation à concevoir. Il se renseigne sur la spécificité du domaine professionnel (les situations de communication, les tâches professionnelles, les textes et discours) tout en décelant les besoins de formation qui en découlent et des supports dont il peut avoir besoin afin d'élaborer et d'assurer les cours commandités. De plus, la découverte du terrain professionnel de référence s'avère décisive pour l'élaboration des activités pédagogiques qui doivent correspondre à la fois aux visées de la formation et à la logique des tâches langagières que le public en formation devra accomplir au travail.

\section{ZONES DE TURBULENCE DANS LA PROGRAMMATION DES FORMATIONS EN LANGUES DE SPÉCIALITÉ}

L'élaboration de la formation en LOS met en relief un type particulier de savoir et savoir-faire que l'enseignant met à profit au cours de la réalisation de ses tâches de concepteur. Selon le cas, ces connaissances et compétences résultent plus ou moins de son expérience professionnelle et pédagogique, de sa formation initiale et/ou continue, de son travail autodidacte. Si les savoirs et les savoir-faire relatifs à la langue et au domaine de spécialité s'avèrent ici pertinents, nous tenons à insister sur les compétences en didac- 
tique sans lesquelles l'enseignant n'est point capable de mettre en place un programme d'enseignement ni d'assurer avec succès sa réalisation ${ }^{2}$. Abordées de manière générale, les étapes de l'élaboration de la formation en langues sur objectifs spécifiques se montrent compréhensibles, évidentes et n'éveillent pas de doutes. Cependant, le diable se cache dans les détails... Il n'est pas rare que les enseignants/ concepteurs aient du mal à mettre en pratique des tâches recommandées par le modèle cité en référence (Mangiante \& Parpette 2004). Ce qui paraissait évident et clair devient source de nombreux points d'interrogation auxquels il convient d'apporter une réponse pertinente. Reste à constater que toutes les étapes du travail conceptuel de l'enseignant comportent les zones de turbulence que nous chercherons à expliciter dans ce qui suit.

\subsection{Analyse de la demande}

Analyser la demande consiste à reconnaître si cette dernière s'inscrit dans la logique de la demande ou dans celle de l'offre (Mangiante \& Parpette 2004). Autrement dit, il importe d'identifier si la formation est commanditée par un public réellement existant et concret (les formations demandées et sur mesure) ou bien si elle est élaborée en tant qu'offre faisant partie de la gamme des formations proposées par un établissement de formation sans être en rapport avec un public précis (les formations offertes). Une telle reconnaissance initiale permet à l'enseignant de programmer la formation en orientant ses contenus vers un degré de spécialisation plus ou moins élevé et vers un profil des apprenants plus ou moins hétéro- / homogène.

De plus, programmer une formation demandée contraint à identifier précisément son vrai bénéficiaire. Dans bien de cas, le commanditaire de la formation et le participant à celle-ci ne correspondent pas à la même personne (p.ex. les formations sollicitées par les employeurs pour leurs salariés). Les intérêts du commanditaire ne coïncident pas toujours avec les intérêts des vrais bénéficiaires de la formation. De plus, l'enseignant / concepteur peut aussi y découvrir une disproportion importante entre les besoins, les motifs et les attentes formulés de deux côtés (Mourlhon-Dallies 2008 ; Carras 2017).

Concevoir la formation en LOS nécessite aussi d'envisager la relation entre l'enseignant et l'apprenant dans une perspective plus large, voire comme

\footnotetext{
${ }^{2}$ Nous avons pu détecter les déficits en compétences didactiques influençant négativement l'élaboration d'un programme d'enseignement au cours de la formation des futurs enseignants FOS. Les problèmes identifiés ont été présentés et discutés dans nos publications antérieures (Sowa 2016 ; 2017).
} 
éléments d'un système plus complexe et conditionnés par un ensemble de divers paramètres (personnels, matériels, situationnels). Chaque situation pédagogique, y compris celle d'enseignement / apprentissage de la langue de spécialité, peut être considérée comme une sorte de système régi par les règles d'organisation et de fonctionnement qui lui sont propres. Influencé par l'environnement (institutionnel, social, culturel, politique, économique) dans lequel il est enraciné, ce système se compose d'éléments relatifs au produit de la formation (le niveau final ou intermédiaire de la langue), aux savoirs et savoir-faire des apprenants présents à l'entrée et à la sortie de la situation pédagogique, aux ressources (matérielles et humaines), contraintes, stratégies, modalités d'évaluation et de rétroaction. Une telle analyse systémique (Eurin Balmet \& Henao de Legge 1992) peut s'avérer utile à l'enseignant / concepteur face à l'analyse de la demande afin de repérer les éléments-clés du système dont il faut obligatoirement tenir compte au cours de la formation et de les séparer des éléments secondaires que l'on peut supprimer du programme. La reconnaissance réussie de tels éléments du système permet $\mathrm{d}$ 'orienter correctement les actions pédagogiques tout en ayant conscience des motifs de la formation demandée.

\subsection{Analyse des besoins}

$S^{\prime}$ interroger sur les besoins contraint à distinguer les besoins subjectifs et ressentis par l'apprenant de ceux qui peuvent être aussi déterminés par d'autres personnes (p.ex. l'enseignant, le directeur de l'établissement scolaire, l'employeur, les institutions gouvernementales etc.). Chacune des personnes susmentionnées peut se faire une autre idée des "besoins », les définir différemment et les orienter vers des objectifs dissemblables. Tout en réclamant la formation linguistique pour ses salariés, l'employeur formule ses propres attentes par rapport au programme d'enseignement, qui peuvent être en décalage avec les besoins, manques, déficits éprouvés par les salariés qui fréquenteront réellement la formation dispensée. L'enseignant / concepteur est alors amené à se demander comment satisfaire aux besoins évoqués par les deux parties. Et qui plus est, les besoins formulés par les apprenants ne doivent pas forcément être identiques à leurs réels besoins langagiers (Hutchinson \& Waters 1987), ce qui contraint l'enseignant à établir un diagnostic de besoins assez détaillé et pertinent.

La diversité de profils professionnels des publics cibles ne facilite point à l'enseignant sa tâche de détermination des besoins. Le problème de l'hétérogénéité des groupes d'apprenants (et donc de leurs besoins) concerne principalement les formations offertes qui réunissent les professionnels 
(actuels ou futurs) appartenant au même domaine de spécialité mais exécutant différents métiers. Cependant, même les groupes professionnellement homogènes au premier abord ne garantissent pas l'identité des besoins de tous les participants de la formation (cf. Mangiante \& Parpette 2004 ; Gajewska \& Sowa 2014).

Les besoins des apprenants concernent principalement le développement / perfectionnement des connaissances / compétences en langue et des aptitudes communicatives par rapport à l'accomplissement des tâches au travail. Cependant, l'enseignant ne devrait pas ignorer ou minimiser les besoins d'apprentissage qui concernent le processus d'apprentissage, la spécificité de la situation pédagogique, les caractéristiques des apprenants, certains aspects culturels et interculturels (Gajewska \& Sowa 2014) qui peuvent séparément ou ensemble - s'avérer importants pour la planification de la formation.

Les aspects techniques de cette étape pèsent également de tout leur poids sur la réussite de l'analyse des besoins. Tout d'abord, l'enseignant / concepteur est confronté à la question de savoir comment identifier les besoins. Certaines situations lui donnent la possibilité de contacter le public avant la formation. Au cours des entretiens directs et à l'aide des questions pointues, il peut alors réunir des informations nécessaires pour déterminer les contenus à aborder et capacités à développer au cours de la formation. Cependant, il y a des situations où un tel contact est exclu. L'enseignant est alors amené à chercher lui-même les sources d'information. La coopération avec le terrain professionnel, la lecture des ouvrages de spécialité, l'analyse des inventaires de compétences apportent certainement de nombreuses informations fort utiles. Il importe cependant d'être conscient du fait que les choix méthodologiques de l'enseignant par rapport à l'analyse des besoins ne restent pas sans influence sur la neutralité de celle-ci : les catégories ou formulations utilisées dans le formulaire d'enquête ou l'interview déterminent déjà une certaine conception de la langue et/ou la théorie de son appropriation (West 1994).

Le moment de l'analyse des besoins constitue aussi un paramètre dont l'enseignant / concepteur devrait tenir compte. En tant qu'élément-clé de la programmation de la formation, l'analyse des besoins apparaît comme l'une des premières tâches accomplies par l'enseignant. Il ne faut pas pourtant oublier sa forte corrélation avec les étapes suivantes de la programmation telles que la collecte des données et l'élaboration des activités, de même qu'avec l'évaluation des résultats des apprenants. Si un programme de formation efficace se base sur une analyse des besoins pertinente, cette dernière s'avère déjà être une forme d'évaluation en elle-même (Frendo 2005). 
L'emplacement de l'analyse des besoins au départ de la programmation des cours de LOS ne signifie aucunement qu'elle est accomplie au début et une fois pour toutes. L'analyse devrait être considérée en revanche de manière évolutive et menée à différents moments de la formation. Il arrive que les besoins des apprenants changent progressivement au cours de la formation ou que ces derniers remettent en question certains choix de l'enseignant, ce qui oblige l'enseignant à rectifier ses décisions une fois la formation commencée. De plus, certains aspects abordés lors des cours peuvent être à l'origine de nouveaux besoins que l'enseignant devra identifier et satisfaire progressivement.

Il convient aussi de mentionner que, lors de l'analyse des besoins de ses apprenants, l'enseignant / concepteur constate ses propres besoins par rapport au programme d'enseignement qu'il doit construire. Le diagnostic des besoins sous forme d'un simple inventaire de situations de communication et d'échanges verbaux ayant lieu en milieu professionnel lui permet d'observer certains déficits par rapport à son propre savoir et/ou savoirfaire (linguistique, professionnel, culturel, etc.). En fonction des déficits de compétences constatés, l'enseignant peut entreprendre certaines démarches d'autoformation et de perfectionnement visant à combler ses lacunes afin de satisfaire aux exigences de la formation et des personnes que cette dernière engage (commanditaires, apprenants, supérieurs). La nature et la taille des besoins concernant l'enseignant sont souvent décisives pour que celui-ci se décide à concevoir et/ou assurer la formation en question, ses lacunes dans le(s) domaine(s) concerné(s) ou ses déficiences méthodologiques pouvant être à la source de son refus.

\subsection{Collecte des donnees}

La pénurie de matériel pédagogique tout prêt pour enseigner la langue à divers publics professionnels contraint l'enseignant à chercher individuellement les sources d'information pouvant être exploitées en vue de la préparation des cours de langue. L'idée de départ, optimiste, selon laquelle il suffirait de demander à une entreprise de pouvoir accéder à sa documentation, d'interviewer et/ou de filmer ses salariés lors de leur travail peut s'avérer difficile voire impossible à réaliser en pratique. Tout d'abord, étant donné que certains documents contiennent des données personnelles, des secrets commerciaux et des informations confidentielles, ils ne peuvent pas être mis à la disposition de tiers. En outre, il n'est pas rare que les entreprises refusent aussi d'enregistrer les professionnels dans leur lieu de travail, même si elles-mêmes sont commanditaires de la formation (cf. Carras 2015). L'enseig- 
nant est alors obligé de chercher des données alternatives devant lui permettre de mettre en œuvre la formation sollicitée.

$\mathrm{Si}$, par chance, l'enseignant / concepteur obtient l'autorisation d'une entreprise à accéder aux discours des professionnels visés, la collecte des données l'oblige à prendre en considération divers paramètres aptes à rendre ces données pertinentes et adéquates par rapport à la formation envisagée. Il importe de rappeler qu'il existe des discours fortement rattachés au contexte et à l'action et d'autres, qui présentent une plus grande autonomie par rapport à la situation dans laquelle ils prennent forme. Autrement dit, la relation entre le discours et le contexte permet d'évaluer le degré d'impact que les facteurs temporels, situationnels et actionnels peuvent avoir sur le discours (cf. contexte fort vs contexte faible ; Hall 1983). Pour ce qui est des contextes forts, « isoler le discours du lieu où il est produit le vide largement de sa signification opérationnelle » (Parpette \& Medina-Jaouen 2015 : 5), parce que la bonne compréhension d'un tel discours ne résulte pas de la seule composante linguistique. Le lieu, le temps et l'action conditionnent fortement la signification des énoncés (p.ex. les échanges entre archéologues sur un chantier des fouilles). Il en va autrement avec les situations à contexte faible (tels le cours magistral ou la conférence de presse) où la captation du discours se réalise avant tout par les éléments langagiers. La maîtrise de telles situations passe donc intégralement par la connaissance de la langue. Il en résulte que la collecte des données doit mettre en avant la dimension discursive et la dimension actionnelle des échanges professionnels produits. Exploiter des données fortement liées au contexte sans faire état de ce contexte les prive de sens et les rend inutiles du point de vue des objectifs de la formation.

Mis à part les données du terrain qui existent en tant que telles et qui sont directement observables et enregistrables (sous forme écrite, audio, vidéo), l'enseignant / concepteur peut être aussi amené à réunir certaines informations au cours des interviews et des demandes de renseignement. Il procède alors à la collecte des données sollicitées, c'est-à-dire "des discours qui seront préparés à l'avance par le concepteur en collaboration avec un auteur de terrain » (Abou Haidar \& Stauber 2015 : 51). De telles données sont complémentaires des données existantes et elles permettent d'expliciter la signification de ces dernières. «Un professionnel peut être filmé en train de travailler mais il n'explique pas nécessairement ce qu'il est en train de faire, comment et pourquoi il agit ainsi. Le discours sollicité permet $\mathrm{d}^{\prime}$ obtenir ces informations qui relèvent de la culture implicite du milieu professionnel » (Abou Haidar \& Stauber 2015 : 51). Étant donné les avantages ${ }^{3}$

${ }^{3}$ Parmi les avantages, il est possible d'énumérer, entre autres, la minimalisation des contraintes du recueil et la souplesse de l'organistion, puisque l'enseignant a la possibilité de 
incontestables des données sollicitées pour l'enseignant, ce dernier doit avoir conscience qu'elles sont créées à sa propre initiative alors que les données existantes reflètent les situations professionnelles à l'état naturel. Si ces dernières présentent le discours dans l'action, les données sollicitées mettent en avant le discours sur l'action. L'infirmière qui parle de ses tâches professionnelles ne formule pas ses énoncés de la même manière lorsqu'elle est en interaction avec le patient ou le médecin. Le passage d'un type de données à l'autre entraîne une transformation, tout en déplaçant des discours pendant l'action vers des discours sur l'action (Parpette \& Medina-Jaouen 2015).

De plus, indispensable pour l'élaboration des formations, la collecte des données pose à l'enseignant d'autres problèmes. Statiques et fixes, les objets se prêtent à être filmés et/ou photographiés sans difficulté. La situation n'est pas la même si l'enseignant est confronté aux paramètres de l'espace et du temps (la durée et la séquentialité des données, l'opportunité de la collecte). Beaucoup d'activités professionnelles s'étendent dans des espaces larges, où les gestes et les paroles d'un professionnel sont liés à ceux d'un autre professionnel situé plus loin (différentes postes dans les fouilles archéologiques) ou plus haut / bas (différentes étages dans le chantier de construction). Les discours professionnels se caractérisent aussi par leur enracinement dans le temps et leur durée ce qui contraint l'enseignant à s'interroger sur leurs limites : où situer le début et la fin d'un discours? comment segmenter les discours longs afin que leur utilisation pédagogique soit possible? Vient finalement la question du bon moment pour recueillir ces données, ce qui amène l'enseignant à résoudre les problèmes de la disponibilité des acteurs interviewés, la possibilité d'accès au terrain professionnel, la répétitivité (ou non) et la prévisibilité (ou non) des situations propices à la collecte, etc.

La présente réflexion sur la collecte des données nous amène enfin à constater que l'enseignant n'est pas toujours suffisamment conscient de la diversité des données environnantes et auxquelles il peut recourir afin de construire d'abord et de réaliser ensuite son programme d'enseignement. Selon Abou Haidar et Stauber (2015), il est possible de classer les données à l'appui de critères tels que le canal (données textuelles, iconiques, audio-visuelles, orales, numériques), le contexte (données finies / infinies, implicites / explicites, concrètes / abstraites, autonomes / indépendantes, ritualisées / non ritualisées), la perspective didactique (données primaires / secondaires,

les refaire si nécessaire. De plus, le contenu linguistique qu'elles comportent est moins complexe et la durée des discours controlable, voire voulue. De par leur caractère ciblé, elles sont utilisables directement en classe. 
manipulables / non manipulables, didactisables / non didactisables) et le discours (discours ritualisés ou non, existants / sollicités / reconstitués, genres discursifs et types d'interaction). La prise en compte de cette variété de données peut s'avérer utile à l'enseignant dans leur exploitation pédagogique puisque le type de données collectées conditionne le discours de l'enseignant sur ces données (Mangiante \& Parpette 2004).

\section{4. Élaboration des activités pédagogiques}

Une fois les données collectées, l'enseignant les soumet à une analyse objective en fonction de leur utilité par rapport aux besoins et finalités de la formation envisagée. Il est alors confronté à la décision sur la manière de mettre ces données au service de la formation. Quelles données peuvent ou doivent être exploitées (entièrement ou partiellement), complétées, modifiées, rejetées etc. ? A partir des données qu'il jugera les mieux adaptées aux objectifs du programme, il élaborera des activités pédagogiques susceptibles de développer la compétence de communication et/ou la connaissance des ressources linguistiques. La structure et le contenu de ces activités restent en relation étroite avec les besoins des apprenants et les finalités espérées de la formation. De plus, ils témoignent également des stratégies d'enseignement retenues. Nous en déduisons qu'il est impossible de détacher l'étape de l'ingénierie pédagogique des décisions prises aux étapes antérieures de la planification (et notamment l'analyse des besoins et celle des données; cf. sections 2.2.-2.3.).

Tout en concevant les types d'activités à exploiter en classe, l'enseignant recourt en principe au même répertoire de techniques qu'utilise son collègue enseignant la langue générale. La différence cruciale entre les activités pédagogiques utilisées au sein des formations sur objectifs ou généraux ne réside pas dans leur forme, mais dans leur finalité. Les formations LOS visent à l'usage de la langue au profit de l'action professionnelle. Il en résulte que dans les activités que l'enseignement des LOS met en place, la langue ne constitue que l'un des instruments du travail. L'interdépendance de la langue et de l'action contraint l'enseignant à concevoir les activités de manière à ce qu'elles soient le plus étroitement liées au contexte professionnel de référence et aux tâches authentiques exécutées par les professionnels dans ce contexte.

On constate, les phases successives de la planification de la formation donnent la priorité à l'activité de l'enseignant alors que les cours de langue en classe sont avant tout «le champ d'action des apprenants » (Mangiante \& Parpette 2004 : 79). L'élaboration des activités exige donc la prise en compte 
de formes de travail qui privilégieront en classe l'activité des apprenants et le maximum de leur apport, tout en rendant la présence de l'enseignant plus discrète et moins imposante. La difficulté de cette étape consiste non seulement à savoir choisir des contenus et les intégrer dans des formes particulières d'activités, mais aussi à savoir réduire le degré d'intervention de l'enseignant pour rendre le travail des apprenants plus autonome et efficace.

\section{CONCLUSION}

Les tâches de l'enseignant et les aspects méthodologiques qu'elles entraînent nous amènent à constater qu'enseigner une langue sur objectifs professionnels passe par les savoirs et savoir-faire pédagogiques particuliers. $C^{\prime}$ est pourquoi nous trouvons particulièrement important d'intégrer les modules de didactique des langues de spécialité dans les programmes de formation des enseignants qu'elle soit initiale ou continue. Concevoir la formation en LOS ne se limite point à une simple sélection des contenus qui seront ensuite coordonnés avec les activités élaborées à l'abri de son bureau et sans rapport avec le milieu du travail. La planification de ce type de formation exige de l'enseignant l'abandon des savoir-faire routiniers et l'oblige à prendre contact et coopérer avec le terrain professionnel externe. En outre, c'est un travail qui requiert une prise d'initiative, de la disponibilité, la volonté $\mathrm{d}$ 'engagement et de coopération. La sensibilisation aux aspects susmentionnés du travail de planification des cours de LOS dans le cadre de la formation initiale prépare mieux à affronter - consciemment et avec plus de succès - les défis méthodologiques du métier d'enseignant.

\section{RÉFÉRENCES}

Abou Haidar, L. / Stauber, J. (2015). Typologie des données en FOS. Points Communs, 2, 44-53.

Carras, C. (2015). Les stratégies de collecte des données : aspects institutionnels et déontologiques. Points Communs, 2, 20-28.

Carras, C. (2017). Le contrat didactique dans les formations en langues sur objectifs spécifiques : quels ajustements face à la multiplicité des acteurs? In: M. Sowa / J. Krajka (eds.), Innovations in languages for specific purposes. Present challenges and future promises (pp. 65-81). Frankfurt am Main: Peter Lang.

Eurin Balmet, S. / Henao de Legge, M. (1992). Pratiques du français scientifique. Paris : Hachette. Frendo, E. (2005). How to teach business English. Harlow : Longman.

Gajewska, E. / Sowa, M. (2014). LSP, FOS, Fachsprache... Dydaktyka języków specjalistycznych. Lublin : Werset.

Grucza, S. (2007). Glottodydaktyka specjalistyczna. Część I. Założenia lingwistyczne dydaktyki języków specjalistycznych. Przegląd Glottodydaktyczny, 23, 7-20. 
Hall, E. T. (1983). The Dance of Life. New York : Anchor Books.

Hutchinson, T. / Waters, A. (1987). English for specific purposes. A learning-centered approach. Cambridge: CUP.

Mangiante, J.-M. / Parpette, Ch. (2004). Le Français sur Objectif Spécifique : de l'analyse des besoins à l'élaboration d'un cours. Paris : Hachette.

Mourlhon-Dallies, F. (2008). Enseigner une langue à des fins professionnelles. Paris : Didier.

Parpette, Ch. / Medina-Jaouen, C. (2015). Les combinaisons entre discours et action : impacts sur la collecte des données et sur les dispositifs de formation linguistique. Points Communs, $2,5-19$.

Sowa, M. (2016). Planowanie kursu języka specjalistycznego: problemy i wyzwania metodyczne przyszłych nauczycieli języka francuskiego dla potrzeb zawodowych. Lingwistyka sStosowana, 19 (4), 119-135.

Sowa, M. (2017). Analyse des besoins au service de l'enseignement des langues de spécialité. Défis et pièges diagnostiques. Acta Philologica, 50, 127-137.

West, R. (1994). Needs analysis in language teaching. Language Teaching, 27, 1-19.

Received: 28.03.2018; revised: 28.01.2019 